\title{
The ubiquitous XP commutator
}

\author{
Alberto C. de la Torre \\ Departamento de Física, Universidad Nacional de Mar del Plata \\ Funes 3350, 7600 Mar del Plata, Argentina \\ dltorre@mdp.edu.ar
}

\begin{abstract}
Position and momentum of a particle can take any value in a continuous spectrum; these values are independent but their indeterminacies are correlated; momentum and position are mutually the generators of the transformations in each other. It is shown in a concise way, how all these features arise solely from their commutation relation $[X, P]=i \hbar$. The article is complete and self contained, adequate for didactic use.
\end{abstract}

\section{INTRODUCTION}

According to quantum mechanics there are four outstanding features concerning the observables of position $X$ and momentum $P$ of a particle. They are:

- The spectrum of position and momentum operators is continuous and can take any real value. That is, physically, the result of measuring position or momentum of a particle can assume any real value. This is of course not surprising since it is also true in classical mechanics, however it is not a trivial statement because in quantum mechanics there are many observables with non continuous, that is, discrete spectra.

- Position and momentum in quantum mechanics, as well as in classical mechanics, are independent. Physically, this means that at a given position of a particle, any value of momentum is possible without any preference, and viceversa, a given momentum can be realized at any position. In classical mechanics we take then two independent variables to describe position and momentum and all other relevant observables are 
functions of these two independent variables. In quantum mechanics this independence must be formalized in a more subtle way: to have a particle at a given position $x$ means that the system is described by a state $\varphi_{x}$, an eigenvector of the position operator $X$ corresponding to the eigenvalue $x$, that is, $X \varphi_{x}=x \varphi_{x}$. If, in this state, any value $p$ of momentum is equally probable, then the probability $\left|\left\langle\varphi_{x}, \phi_{p}\right\rangle\right|^{2}$ must be independent of $p$, where $\phi_{p}$ is the eigenvector of the momentum operator associated to the eigenvalue $p$, that is, $P \phi_{p}=p \phi_{p}$. Similarly, if the particle has some value of momentum $p$, its state is $\phi_{p}$ and in order to have any position $x$ equally probable we must have $\left|\left\langle\phi_{p}, \varphi_{x}\right\rangle\right|^{2}$ independent of $x$. Now the two sets $\left\{\varphi_{x}, \forall x\right\}$ and $\left\{\phi_{p}, \forall p\right\}$ are two different bases of the Hilbert space and they are called mutually unbiased if the number $\left|\left\langle\varphi_{x}, \phi_{p}\right\rangle\right|$ is a constant independent of $x$ and $p$. Therefore the independence of position and momentum in quantum mechanics is formalized by the requirement that their corresponding bases should be unbiased.

- The values of position and momentum are independent but their indeterminacies in any state $\psi$, given by $\Delta_{x}^{2}=\left\langle\psi,(X-\langle X\rangle)^{2} \psi\right\rangle$ and $\Delta_{p}^{2}=\left\langle\psi,(P-\langle P\rangle)^{2} \psi\right\rangle$, where $\langle X\rangle=$ $\langle\psi, X \psi\rangle$ and $\langle P\rangle=\langle\psi, P \psi\rangle$ are the expectation values of position and momentum, are correlated such that $\Delta_{x} \Delta_{p} \geq \hbar / 2$. This feature is unique to quantum mechanics since there are no indeterminacies in classical mechanics. The correlation manifest in the uncertainty principle is unavoidable and appears in all states; however for some states, the lower bound in the inequality can take a larger value as we will see later.

- Momentum and position observables are generators of translations in space and of increases in momentum. This relation, also present in classical mechanics, is formalized in quantum mechanics by the definition of a unitary operator $U_{a}=\exp \left(-\frac{i}{\hbar} a P\right)$ that performs the transformations of states and operators in the Hilbert space corresponding to the translations in physical space $X \rightarrow X+a$. So for any function of the position operator we have $F(X+a)=U_{a}^{\dagger} F(X) U_{a}$. Similarly, the operator $V_{g}=\exp \left(+\frac{i}{\hbar} g X\right)$ describes in the Hilbert space the boosts ${ }^{1} P \rightarrow P+g$, and $G(P+g)=V_{g}^{\dagger} G(P) V_{g}$.

A beautiful feature of the formalism of quantum mechanics is that al properties mentioned above can be shown to be consequences of the commutation relation $[X, P]=i \hbar$ 
solely. The commutator is indeed ubiquitous and contains encoded all relevant properties of position, momentum and their relations. We will give a complete proof of the above statements, although some parts are very well known and appear in most textbooks. We do this because in some cases the proof presented here have some advantages compared to the standard ones (for instance of the uncertainty relations) and some other parts of the proof are absent from most textbooks, even from advanced ones.

\section{INDETERMINACY RELATIONS}

Let us start presenting a proof ${ }^{2}$ of the indeterminacy relations in the optimized version given by Schrödinger ${ }^{3}$ three years after the appearance of Heisenberg's uncertainty relations ${ }^{4}$. For this proof, it is convenient to work with the "centered" operators defined as $\widetilde{X}=X-\langle X\rangle$ and $\widetilde{P}=P-\langle P\rangle$ (of course, the expectation values are multiplying an identity operator not shown) and therefore we have, for any state $\psi, \Delta_{x}^{2}=\left\langle\psi, \widetilde{X}^{2} \psi\right\rangle=\langle\widetilde{X} \psi, \widetilde{X} \psi\rangle=\|\widetilde{X} \psi\|^{2}$ and similarly $\Delta_{p}^{2}=\left\langle\psi, \widetilde{P}^{2} \psi\right\rangle=\langle\widetilde{P} \psi, \widetilde{P} \psi\rangle=\|\widetilde{P} \psi\|^{2}$. Their product is then

$$
\Delta_{x}^{2} \Delta_{p}^{2}=(\|\widetilde{X} \psi\|\|\widetilde{P} \psi\|)^{2} \geq|\langle\widetilde{X} \psi, \widetilde{P} \psi\rangle|^{2}=|\langle\psi, \widetilde{X} \widetilde{P} \psi\rangle|^{2}
$$

The inequality sign comes from Cauchy-Schwarz inequality for the internal product $|\langle f, g\rangle| \leq$ $\|f\|\|g\|$. Now we can write the product $\widetilde{X} \widetilde{P}=\frac{1}{2}\{\widetilde{X} \widetilde{P}\}+i \frac{1}{2 i}[\widetilde{X} \widetilde{P}]$. In this way, the product is decomposed in the hermitian part plus the antihermitian part (similar to the decomposition of a complex number in real and imaginary part), and therefore we have

$$
\Delta_{x}^{2} \Delta_{p}^{2} \geq\left(\left\langle\psi, \frac{1}{2}\{\widetilde{X}, \widetilde{P}\} \psi\right\rangle\right)^{2}+\left(\left\langle\psi, \frac{1}{2 i}[\widetilde{X}, \widetilde{P}] \psi\right\rangle\right)^{2}
$$

Now we replace the commutator and anticommutator

$$
\begin{aligned}
{[\widetilde{X}, \widetilde{P}] } & =[X, P]=i \hbar \\
\{\widetilde{X}, \widetilde{P}\} & =\{X, P\}-2 X\langle P\rangle-2\langle X\rangle P+2\langle X\rangle\langle P\rangle,
\end{aligned}
$$

and we finally get the indeterminacy relation

$$
\Delta_{x}^{2} \Delta_{p}^{2} \geq\left(\frac{\hbar}{2}\right)^{2}+\left(\frac{1}{2}\langle\psi,(X P+P X) \psi\rangle-\langle X\rangle\langle P\rangle\right)^{2} .
$$

The second parenthesis vanishes for some states (for instance gaussian packets) and it is ignored in many texts because it is nonnegative. However, for other states, it gives an inequality more restrictive than the original Heisenberg's relation $\Delta_{x} \Delta_{p} \geq \hbar / 2$. 


\section{GENERATORS}

We will now prove that the commutation relation implies that the momentum $P$ is the generator of translations $X \rightarrow X+a$. Precisely, we will show that if $[X, P]=i \hbar$ then for any function (that can be expanded as a power series) $F(X)$ it is

$$
F(X+a)=U_{a}^{\dagger} F(X) U_{a}
$$

where the unitary operator $U_{a}$ is given by

$$
U_{a}=\exp \left(-\frac{i}{\hbar} a P\right)
$$

and if $\varphi_{x}$ is an eigenvector of the position operator $X$ corresponding to the eigenvalue $x$, then $U_{a} \varphi_{x}$ is an eigenvector corresponding to the eigenvalue $x+a$.

In order to prove Eq.(6) we will consider the Taylor series expansion of both sides of the equation. The left hand side will be expanded as a function of $X$ and the expansion will involve $n$th order derivatives of $F(X)$, whereas the right hand side will be expanded as a function of the parameter $a$ and it will involve derivatives with respect to $a$ that, due to the rule for the derivative of a product, will be given by an $n$th order commutator. The proof is obtained by the identification of the derivatives of $F(X)$ with its commutator with $P$. Let us begin by this last step: If $[X, P]=i \hbar$, then for any function $F(X)$ (that can be expanded as a power series) it is

$$
\frac{d^{n} F(X)}{d X^{n}}=\frac{-i}{\hbar}\left[\frac{-i}{\hbar}\left[\cdots \frac{-i}{\hbar}[F(X), P], P\right], \cdots, P\right]=\left(\frac{-i}{\hbar}[)^{n} F(X)(, P]\right)^{n}
$$

where the " $n$ exponent" means an $n$ fold repetition of the enclosed symbols. In order to prove this we first notice that

$$
\left[X^{n}, P\right]=i \hbar n X^{n-1}=\frac{d}{d X} i \hbar X^{n}
$$

that can be proven by mathematical induction: for $n=1$ it is valid, assuming that it is valid for $n$, we prove that it is valid for $n+1$. Indeed $\left[X^{n+1}, P\right]=X\left[X^{n}, P\right]+[X, P] X^{n}=$ $X i \hbar n X^{n-1}+i \hbar X^{n}=i \hbar(n+1) X^{n}$. Now we take an arbitrary set of coefficients $\left\{C_{n}\right\}$ to multiply Eq.(9) and sum over $n$. So we get for any function $F(X)=\sum_{n} C_{n} X^{n}$

$$
[F(X), P]=i \hbar \frac{d F(X)}{d X} .
$$


If we iterate this result, for the $n$th order derivative we obtain Eq.(8).

Let us consider now he right hand side of Eq.(6) as a function of $a$ and its derivatives.

$$
\begin{aligned}
f(a) & =\exp \left(\frac{i}{\hbar} a P\right) F(X) \exp \left(\frac{-i}{\hbar} a P\right) \\
\frac{d f(a)}{d a} & =\frac{i}{\hbar} P f(a)+f(a) \frac{-i}{\hbar} P=\frac{-i}{\hbar}[f(a), P] \\
\frac{d^{2} f(a)}{d a^{2}} & =\frac{-i}{\hbar}\left[\frac{-i}{\hbar}[f(a), P], P\right] \\
\ldots \ldots & \ldots \\
\frac{d^{n} f(a)}{d a^{n}} & =\left(\frac{-i}{\hbar}[)^{n} f(a)(, P]\right)^{n} .
\end{aligned}
$$

With these derivatives, evaluated at $a=0$, we obtain the Taylor expansion

$$
f(a)=\left.\sum_{n=0}^{\infty} \frac{a^{n}}{n !} \frac{d^{n} f(a)}{d a^{n}}\right|_{a=0}=\sum_{n=0}^{\infty} \frac{a^{n}}{n !}\left(\frac{-i}{\hbar}[)^{n} F(X)(, P]\right)^{n} .
$$

But now, from Eq.(8) the $n$th order commutator is replaced by the $n$th order derivative of $F(X)$ and we obtain precisely the Taylor expansion of $F(X+a)$ :

$$
f(a)=\sum_{n=0}^{\infty} \frac{a^{n}}{n !} \frac{d^{n} F(X)}{d X^{n}}=F(X+a) .
$$

This completes the proof of Eq.(6). As a particular case of this result, when the function $F(X)$ is the identity, we have $X+a=U_{a}^{\dagger} X U_{a}$ and performing a left-product with $U_{a}$ we obtain the commutator

$$
\left[X, U_{a}\right]=a U_{a}
$$

With this commutator we can easily prove that $U_{a}$ is a shift operator for the eigenvectors of the position operator.

$$
X U_{a} \varphi_{x}=U_{a} X \varphi_{x}+a U_{a} \varphi_{x}=(x+a) U_{a} \varphi_{x}
$$

therefore (assuming nondegeneracy)

$$
U_{a} \varphi_{x}=\varphi_{x+a}
$$

We have proven that $P$ is the generator of translations $X \rightarrow X+a$ and exactly in the same way we can prove that $X$ is the generator of boosts $P \rightarrow P+g$ with the difference 
in the sign of the exponent in the unitary operator $V_{g}=\exp \left(+\frac{i}{\hbar} g X\right)$. Then we have $G(P+g)=V_{g}^{\dagger} G(P) V_{g}$, and if $\phi_{p}$ is an eigenvector of the position operator $P$ corresponding to the eigenvalue $p$, then $V_{g} \phi_{p}=\phi_{p+g}$ is an eigenvector corresponding to the eigenvalue $p+g$.

Another interesting consequence or Eq.(6) follows from the special case where we take $F(X)=V_{g}=\exp \left(+\frac{i}{\hbar} g X\right)$. With this we get: $\exp \left(\frac{i}{\hbar} g a\right) V_{g}=U_{a}^{\dagger} V_{g} U_{a}$, and from this we obtain the commutation relation for the unitary transformations corresponding to translations and boosts

$$
\left[V_{g}, U_{a}\right]=\left(\exp \left(\frac{i}{\hbar} g a\right)-1\right) U_{a} V_{g}
$$

Although $X$ and $P$ do not commute, boosts and translations commute when the parameters are such that $a g=2 \pi \hbar m$ for any integer $m$. This interesting relation was derived for solid state physics ${ }^{5}$ where $a$ is a lattice constant and $g$ is the reciprocal lattice constant.

\section{CONTINUITY AND INDEPENDENCE}

With the results of the previous section it is very easy to prove that the spectra of $X$ and $P$ are continuous and that their bases are unbiased. The continuity follows from the fact that there is no restriction on the value that the parameters $a$ and $g$ can take in $U_{a}$ and $V_{g}$ and therefore they can be infinitesimal. So if $x$ is an eigenvalue for the position operator then $x+\varepsilon$ with $\varepsilon \rightarrow 0$ is also an eigenvalue (similarly for momentum eigenvalues). In order to see that the basis $\left\{\varphi_{x}\right\}$ associated to $X$ and $\left\{\phi_{p}\right\}$ associated to $P$ are unbiased we can first prove that $\left|\left\langle\varphi_{x}, \phi_{p}\right\rangle\right|=\left|\left\langle\varphi_{x+s}, \phi_{p}\right\rangle\right|$ for any $s$, that is, $\left|\left\langle\varphi_{x}, \phi_{p}\right\rangle\right|$ is independent of $x$. Indeed we have $\left|\left\langle\varphi_{x}, \phi_{p}\right\rangle\right|=\left|e^{\frac{i}{\hbar} s p}\left\langle\varphi_{x}, \phi_{p}\right\rangle\right|=\left|\left\langle\varphi_{x}, e^{\frac{i}{\hbar} s p} \phi_{p}\right\rangle\right|=\left|\left\langle\varphi_{x}, e^{\frac{i}{\hbar} s P} \phi_{p}\right\rangle\right|=\left|\left\langle e^{\frac{-i}{\hbar} s P} \varphi_{x}, \phi_{p}\right\rangle\right|=\left|\left\langle\varphi_{x+s}, \phi_{p}\right\rangle\right|$.

In the same way we see that $\left|\left\langle\varphi_{x}, \phi_{p}\right\rangle\right|$ is independent of $p$ and therefore the bases are mutually unbiased.

Actually, from the commutation relation we can get more than just proving that $\left|\left\langle\varphi_{x}, \phi_{p}\right\rangle\right|$ is constant; we can get the dependence of the complex number $\left\langle\varphi_{x}, \phi_{p}\right\rangle$ on $x$ and $p$, that is, we can calculate the coefficients of the unitary transformation between the two bases. For this, let us consider $\left\langle\varphi_{x+\varepsilon}, \phi_{p}\right\rangle=\left\langle U_{\varepsilon} \varphi_{x}, \phi_{p}\right\rangle=\left\langle\varphi_{x}, U_{\varepsilon}^{\dagger} \phi_{p}\right\rangle=\left\langle\varphi_{x}, \exp \left(\frac{i}{\hbar} \varepsilon P\right) \phi_{p}\right\rangle=$ $\exp \left(\frac{i}{\hbar} \varepsilon p\right)\left\langle\varphi_{x}, \phi_{p}\right\rangle$. With this we can build the derivative

$$
\frac{d\left\langle\varphi_{x}, \phi_{p}\right\rangle}{d x}=\lim _{\varepsilon \rightarrow 0} \frac{\left\langle\varphi_{x+\varepsilon}, \phi_{p}\right\rangle-\left\langle\varphi_{x}, \phi_{p}\right\rangle}{\varepsilon}=\lim _{\varepsilon \rightarrow 0} \frac{\exp \left(\frac{i}{\hbar} \varepsilon p\right)-1}{\varepsilon}\left\langle\varphi_{x}, \phi_{p}\right\rangle=\frac{i}{\hbar} p\left\langle\varphi_{x}, \phi_{p}\right\rangle,
$$


and integrating the equation we get

$$
\left\langle\varphi_{x}, \phi_{p}\right\rangle=K \exp \left(\frac{i}{\hbar} x p\right)
$$

with an arbitrary constant $K$.

\section{CONCLUSION}

We have seen that all essential properties of the position and momentum observables as well as their relations arise in the formalism of quantum mechanics from a unique feature: the commutation relation $[X, P]=i \hbar$. However this fundamental feature is a mathematical abstraction and it is therefore convenient to emphasize its physical consequences. In the teaching of quantum mechanics we can take two options: one can postulate the commutation relations (postulate of canonical quantization) and from this postulate to derive the physical consequences, or, the reverse way, to define first the generators of translations and boosts and derive the commutation relations of the corresponding operators. The present paper is useful for both strategies because it clearly presents the tight relationship between the commutation relations and its physical consequences. For this reason it was decided to present here a complete proof, although some parts of it, but not all of them, can be found spread in several texts.

\section{ACKNOWLEDGMENTS}

This work received partial support from "Consejo Nacional de Investigaciones Científicas y Técnicas" (CONICET), Argentina. 


\section{REFERENCES}

${ }^{1}$ In this work we use the term "boost" with a meaning related, but different from the one used in Galilei transformations where the boosts are $X \rightarrow X+v t$ and their generator is $B=P t-m X$.

${ }^{2}$ This is a simplified version of the proof presented in A. C. de la Torre, P. Catuogno, S. Ferrando. "Uncertainty and nonseparability", Found. of Phys. Lett 2, 235-244 (1989).

${ }^{3}$ E. Schrödinger, "Zum Heisenbergschen Unschärfeprinzip", Sitzungberichte der Preußischen Akademie der Wissenschaft. Physikalische-mathematische Klasse. 296-303, (1930).

${ }^{4}$ W. Heisenberg, "Über den anschaulichen Inhalt der quantentheoretischen Kinematik und Mechanik", Zeitschrift für Physik 43, 172-198 (1927).

${ }^{5}$ J. Zak, "Finite translations in solid-state physics" Phys. Rev. Lett. 19, 1385-1387 (1967). 